\title{
A NEW DECISION MODEL FOR CROSS-DOCKING CENTER LOCATION IN LOGISTICS NETWORKS UNDER INTERVAL-VALUED INTUITIONISTIC FUZZY UNCERTAINTY
}

\author{
Seyed Meysam MOUSAVI ${ }^{1}$, Jurgita ANTUCHEVIČIENË ${ }^{2 *}$, \\ Edmundas Kazimieras ZAVADSKAS ${ }^{3}$, Behnam VAHDANI ${ }^{4}$, Hassan HASHEMI ${ }^{5}$ \\ ${ }^{1}$ Dept of Industrial Engineering, Shahed University, Tehran, Iran \\ ${ }^{2,}{ }^{3}$ Dept of Construction Management and Real Estate, \\ Vilnius Gediminas Technical University, Lithuania \\ ${ }^{4}$ Dept of Industrial Engineering, Qazvin Branch, Islamic Azad University, Qazvin, Iran \\ ${ }^{5}$ School of Civil Engineering, Iran University of Science and Technology, Tehran, Iran
}

Received 29 June 2017; revised 27 August 2017; accepted 1 November 2017

\begin{abstract}
Cross-dock has been a novel logistic approach to effectively consolidate and distribute multiple products in logistics networks. Location selection of cross-docking centers is a decision problem under different conflicting criteria. The decision has a vital part in the strategic design of distribution networks in logistics management. Conventional methods for the location selection of cross-docking centers are insufficient for handling uncertainties in Decision-Makers (DMs) or experts' opinions. This study presents a new Multi-Criteria Group Decision-Making (MCGDM) model, which applies the concept of compromise solution under uncertainty. To address uncertainty, Interval-Valued Intuitionistic Fuzzy (IVIF) sets are used. In this paper, first an IVIF-weighted arithmetic averaging (IVIF-WAA) operator is used in order to aggregate all IVIF-decision matrices, which were made by a team of the DMs into final IVIF-decision matrix. Then, a new Collective Index (CI) is developed that simultaneously regards distances of cross-docking centers as candidates from the IVIF-ideal points. Finally, the feasibility and practicability of proposed MCGDM model is illustrated with an application example on location choices of cross-docking centers to the logistics network design.
\end{abstract}

Keywords: multiple cross-docks, location evaluation and selection, logistics networks, multi-criteria decision-making, group decision process, interval-valued intuitionistic fuzzy sets.

\section{Introduction}

Cross-docking as a modern logistic approach has high potentials to decrease transportation costs as well as delivery time without increasing the inventory. Indeed, cross-docking can be the direct movement of containers from receiving to shipping with minimum dwell time in between. This approach has crucial impacts on the logistics networks, including just-in-time, zero inventories, electronic data exchange and different drop ship methods (Kellar et al. 2016; Mousavi, Vahdani 2017). In the last decade, the approach has been applied to several logistics networks, for instance, Wal-Mart's logistics network and United States' Postal Service with remarkable results (Alkhedher 2006; Yan, Tang 2009; Mousavi et al. 2014a; Khalaj et al. 2014; Maknoon et al. 2016).
In today's competitive environment, appropriate crossdocks location has been taken as the main critical activity for effective distribution systems in the logistics management (Ladier, Alpan 2016). Effective movement of products from suppliers or providers through cross-docking distribution centers to retailers or customers is very important in the logistics networks (Mousavi et al. 2014b). Consequently, the utilization of several cross-docking centers is more and more vital.

In logistics studies, some papers have been published on the location problem of distribution centers and warehouses; for instance, Vlachopoulou et al. (2001) proposed geographic-DSS for the evaluation of warehouse sites to identify the suitable alternative. Lee (2005) presented

${ }^{*}$ Corresponding author. E-mail: jurgita.antucheviciene@vgtu.lt 
fuzzy Multi-Criteria Decision-Making (MCDM) method to solve the distribution center selection problem in a fuzzy environment. Ou and Chou (2009) took evaluation criteria of international distribution centers into account and introduced a fuzzy rating system. Demirel et al. (2010) considered the warehouse location selection under multiple quantitative and qualitative criteria by using Choquet integral. Özcan et al. (2011) compared three MCDM methods and grey theory for the application to the warehouse selection problem.

There are a few papers regarding the optimal crossdock location in the logistics networks. Ratliff et al. (1998) formulated an integer-programming model by allocating the required truckloads to the cross-dock routes to minimize the number of truck miles. Jayaraman and Ross (2003) solved the facility location problem by cross-docks to reduce the costs related to opening facilities and transportation. The parameters regarding the performance of a cross-dock investigated by Bartholdi and Gue (2003), which can be used for designing the cross-dock layout and can be considered as objectives in optimizing the utility of cross-dock facilities. Makui et al. (2006) extended a mathematical programming with multi-objective location allocation for cross-docking centers and used a genetic algorithm as a solving approach. Ross and Jayaraman (2008) assessed some control mechanisms based on their previous study in Jayaraman and Ross (2003), and indicated several experimental convergences.

The review of the cross-docking published papers indicates that all of the above literature applied crisp values (i.e., deterministic model) to descript the experts or Decision-Makers (DMs)' opinions on cross-docking candidates; however, in practice this is seldom the case in the logistics environment. Different types of uncertainty and imprecision (i.e., fuzziness) often are available in the decision-making. Due to these uncertainties in real-life situations, the experts or DMs are often unsure of their preferences during the cross-docking location selection processes. It is mainly because the information, including their preferences over candidates and the relative importance of criteria, is incomplete, uncertain and imprecise. Moreover, decision criteria are often subjective and qualitative in the strategic decision-level, and thus it may be difficult for the DMs to describe judgments about candidates with precise numerical values.

This study takes a new decision-making approach into account under uncertainty to address location problem of cross-docks. To cope with uncertainty, new extension of fuzzy sets, namely Interval-Valued Intuitionistic Fuzzy (IVIF) set, is taken into consideration in this study. In the last two decades, most decision studies under uncertainty considered the traditional fuzzy theory developed by $\mathrm{Za}$ deh (1965). In comparison with Zadeh's fuzzy sets, Intuitionistic Fuzzy Sets (IFSs) proposed by Atanassov (1986) perform well and are more useful to address uncertainty and vagueness. Hence, experts can employ IFSs, particularly IVIF sets introduced by Atanassov and Gargov (1989) to describe the information of candidates versus multi-criteria for decision-making problems under incomplete and uncertain environment. The IVIF sets can effectively consider the uncertainty and fuzziness in information and DMs' judgments (e.g., Atanassov 1994; Xu 2007a, 2007b; Wei, Wang 2007; Park et al. 2011; Hashemi et al. 2014). Recently several multi-criteria decision-making methods were extended for Group Decision-Making (GDM) process problems in an uncertain environment (e.g., Razavi Hajiagha et al. 2013; Zavadskas et al. 2014, 2015, 2018a, 2018b; Chen 2015, 2016; Xue et al. 2016; Prentkovskis et al. 2018; Hashemi et al. 2016; Kahraman et al. 2017; Stević et al. 2017). A number of Multi-Criteria Group Decision-Making (MCGDM) methods based on intuitionistic fuzzy operators were suggested (Rong et al. 2016; Liu et al. 2016; Li et al. 2016), a linear programming technique for MCGDM problems with IVIF information was evolved (Razavi Hajiagha et al. 2015). Taking into account the increased interest, a special issue on IFS theory and its application in economy, technology and management was published (Liu 2016).

This study presents a novel Interval-Valued Intuitionistic Fuzzy Multi-Criteria Group Decision-Making (IVIFMCGDM) model by the concept of compromise solution - i.e., Technique for Order Performance by Similarity to Ideal Solution (TOPSIS) method - to handle the CrossDocking Centers Location Selection Problem (CDLSP), under uncertainty. Characteristics of candidates and decision criteria are determined by linguistic terms and then represented by IVIF-numbers through the GDM. The model creates more generality and flexibility to express the uncertainties according to a lack of data in the traditional decision methods. Furthermore, the model allows a team of DMs to express their degrees of membership in addition to their degree of non-membership in interval form while evaluating the weight of each criterion in the GDM process.

In the IVIF-MCGDM model, in order to address the fuzziness of decision-making process, linguistic variables are applied. The information of the process is addressed by using IVIF-decision matrices. The Interval-Valued Intuitionistic Fuzzy Weighted Arithmetic Averaging (IVIF-WAA) operator is applied to aggregate all experts' opinions for the performance values of cross-docks as candidates as well as the weight of each criterion. Then, two new basic IVIF-operations, namely subtraction and division, are presented to construct IVIF-ideal matrices in the assessment process. Finally, a new index is developed to rank each cross-docking center (candidate), which is based on a score function to measure IVIF-numbers and distances from IVIF-ideal solutions.

In summary, main novel characteristics of IVIF-MCGDM approach in cross-docking networks are as follows:

- proposing a new MCGDM model based on the concepts of the compromise solution and IVIF sets by employing linguistic terms, in which the performance ratings of each candidate in addition to the weight of each criterion are achieved by IVIF numbers; 
- developing a new ranking index to distinguish candidates in IVIF forms for the ranking purpose by simultaneously constructing IVIF-ideal matrices;

- applying novel operations on the IVIF sets (i.e., subtraction and division operations);

- furthermore, an application example is examined and solved to address the CDLSP in the logistics network design under uncertainty.

The remainder of this paper is organized as follows. In Section 1, the IVIF-MCGDM model is given. In Section 2, an application example for the CDLSP is presented in detail. Discussions of results are presented in Section 3. Conclusions are reported in last section.

\section{Proposed new IVIF-MCGDM model}

\section{Indices and sets:}

$h$ - index of DMs;

$i$ - index of potential candidates;

$j \quad$ - index of evaluation attributes;

$C D$ - set of potential candidates, $C D=\left\{C D_{1}, C D_{2}, \ldots, C D_{m}\right\}$;

$E A$ - set of evaluation attributes, $E A=\left\{E A_{1}, E A_{2}, \ldots, E A_{n}\right\}$;

$T M$ - set of DMs, TM $=\left\{T M_{1}, T M_{2}, \ldots, T M_{l}\right\}$.

\section{Parameters:}

$a_{i j}$ - lower bound of $\bar{\mu}$ of aggregated score $\tilde{x}_{i j}$;

$a_{j}^{\star}$ - lower bound of $\bar{\mu}$ of positive-ideal solution;

$a_{j}^{-}$- lower bound of $\bar{\mu}$ of negative-ideal solution;

$a_{i j}^{(h)}-$ lower bound of $\bar{\mu}$ of score $\tilde{x}_{i j}^{(h)}$;

$b_{i j}$ - upper bound of $\bar{\mu}$ of aggregated score $\tilde{x}_{i j}$;

$b_{j}^{*}$ - upper bound of $\bar{\mu}$ of positive-ideal solution;

$b_{\dot{j}}^{-}$- upper bound of $\bar{\mu}$ of negative-ideal solution;

$b_{i j}^{(h)}$ - upper bound of $\bar{\mu}$ of score $\tilde{x}_{i j}^{(h)}$;

$c_{i j}$ - lower bound of $\bar{v}$ of aggregated score $\tilde{x}_{i j}$;

$c_{j}^{*}$ - lower bound of $\bar{v}$ of positive-ideal solution;

$c_{j}^{-}$- lower bound of $\bar{v}$ of negative-ideal solution;

$c_{i j}^{(h)}$ - lower bound of $\bar{v}$ of score $\tilde{x}_{i j}^{(h)}$;

$d_{i j}$ - upper bound of $\bar{v}$ of aggregated score $\tilde{x}_{i j}$;

$d_{j}^{*}$ - upper bound of $\bar{v}$ of positive-ideal solution;

$d_{j}^{-}$- upper bound of $\bar{v}$ of negative-ideal solution;

$d_{i j}^{(h)}$ - upper bound of $\bar{v}$ of score $\tilde{x}_{i j}^{(h)}$;

$l$ - number of DMs;

$m$ - number of potential candidates;

$n$ - number of evaluation attributes;

$n \tilde{s}_{i j}^{-}$- IVIF-distance between $\tilde{p}_{i j}$ and $\tilde{p}_{j}^{-}$;

$\tilde{p}_{i j}$ - IVIF-weighted aggregated score of the $i$-th potential candidate $C D_{i}$ with respect to the $j$-th evaluation attribute $E A_{j}$;

$\tilde{p}_{j}^{*}$ IVIF-positive-ideal solution;

$\tilde{p}_{j}^{-}$- IVIF-negative-ideal solution; $p \tilde{s}_{i j}^{*}$ - IVIF-distance between $\tilde{p}_{i j}$ and $\tilde{p}_{j}^{*}$;

$\tilde{x}_{i j}$ - IVIF-aggregated score of the $i$-th potential candidate $C D_{i}$ with respect to the $j$-th evaluation attribute $E A_{j}$;

$\tilde{x}_{i j}^{(h)}$ - IVIF-score of the $i$-th potential candidates $C D_{i}$ with respect to the $j$-th evaluation attribute $E A_{j}$ provided by $h$-th DM;

$\tilde{\xi}^{(h)}$ - IVIF-DM's weight of $h$-th expert;

$\bar{\mu}$ - interval value of membership degree of IVIF numbers;

$\bar{v}$ - interval value of non-membership degree of IVIF numbers;

$\tilde{\varpi}_{j}$ - IVIF-aggregated weight of attribute $j$;

$\tilde{\varpi}_{j}^{(h)}$ - IVIF-weight of attribute $j$ provided by $h$-th DM;

$\omega_{j 1}$ - lower bound of $\bar{\mu}$ of aggregated weight $\tilde{\bar{\varpi}}_{j}$;

$\omega_{j 3}$ - lower bound of $\bar{v}$ of aggregated weight $\tilde{\varpi}_{j}$;

$\xi_{1}^{(h)}$ - lower bound of $\bar{\mu}$ of DM's weight $\tilde{\xi}^{(h)}$;

$\xi_{3}^{(h)}$ - lower bound of $\bar{v}$ of DM's weight $\tilde{\xi}^{(h)}$;

$\xi_{2}^{(h)}$ - upper bound of $\bar{\mu}$ of DM's weight $\tilde{\xi}^{(h)}$;

$\xi_{4}^{(h)}$ - upper bound of $\bar{v}$ of DM's weight $\tilde{\xi}^{(h)}$;

$\omega_{j 1}^{(h)}$ - lower bound of $\bar{\mu}$ of weight $\tilde{\varpi}_{j}^{(h)}$;

$\omega_{j 3}^{(k)}$ - lower bound of $\bar{v}$ of weight $\tilde{\boldsymbol{\omega}}_{j}^{(h)}$;

$\omega_{j 2}$ - upper bound of $\bar{\mu}$ of aggregated weight $\tilde{\bar{\sigma}}_{j}$;

$\omega_{j 4}$ - upper bound of $\bar{v}$ of aggregated weight $\tilde{\bar{\sigma}}_{j}$;

$\omega_{j 2}^{(k)}$ - upper bound of $\bar{\mu}$ of weight $\tilde{\boldsymbol{\omega}}_{j}^{(h)}$;

$\omega_{j 4}^{(k)}$ - upper bound of $\bar{v}$ of weight $\tilde{\omega}_{j}^{(h)}$.

\section{Decision variables:}

$C I_{i} \geq 0$ - final ranking index;

$\tilde{\kappa}_{i j^{\prime}} \geq 0$ - max-separation measure of $\eta_{i}$;

$\tilde{\chi}_{i j^{\prime}} \geq 0$ - min-separation measure of $\vartheta_{i}$;

$\eta_{i} \geq 0$ - the first separation measure of final ranking index;

$\vartheta_{i} \geq 0 \quad$ - the second separation measure of final ranking index.

Proposed GDM problem under multi-criteria involves vague and uncertain data in the CDLSP. The characteristic of the candidate $C D_{i}$ is denoted by an IVIF set as follows:

$$
\begin{aligned}
& C D_{i}=\left\{\left\langleE A_{j},\left[\mu^{L}\left(C D_{i}, E A_{j}\right), \mu^{U}\left(C D_{i}, E A_{j}\right)\right],\right.\right. \\
& \left.\left.\left[v^{L}\left(C D_{i}, E A_{j}\right), v^{U}\left(C D_{i}, E A_{j}\right)\right]\right\rangle \mid E A_{j} \in E A\right\},
\end{aligned}
$$

where:

$0 \leq \mu^{U}\left(C D_{i}, E A_{j}\right)+v^{U}\left(C D_{i}, E A_{j}\right) \leq 1 ;$

$\mu^{L}\left(C D_{i}, E A_{j}\right) \geq 0$;

$v^{L}\left(C D_{i}, E A_{j}\right) \geq 0$;

$i=1, \ldots, m$;

$j=1, \ldots, n$. 
IVIF set value that can be a pair of intervals:

$$
\begin{aligned}
& \mu^{(h)}\left(C D_{i}, E A_{j}\right)= \\
& {\left[a_{i j}^{(h)}, b_{i j}^{(h)}\right], v^{(h)}\left(C D_{i}, E A_{j}\right)=\left[c_{i j}^{(h)}, d_{i j}^{(h)}\right]}
\end{aligned}
$$

for $E A_{j} \in E A$ is presented with:

$$
x_{i j}^{(h)}=\left\langle\left[a_{i j}^{(h)}, b_{i j}^{(h)}\right],\left[c_{i j}^{(h)}, d_{i j}^{(h)}\right]\right\rangle,
$$

where: $\left[a_{i j}^{(h)}, b_{i j}^{(h)}\right]$ and $\left[c_{i j}^{(h)}, d_{i j}^{(h)}\right]$ depict the degrees that candidate $C D_{i}$ can satisfy and cannot satisfy the criterion $E A_{j}$ by $\mathrm{DM} \mathrm{TM}_{h}(h=1, \ldots, l)$ respectively.

Then, $X^{(h)}=\left(x_{i j}^{(h)}\right)_{m \times n}$ with DM $\left(T M_{h}\right)$ in form of an IVIF-decision matrix is achieved as below:

$$
X^{(h)}=\left(x_{i j}^{(h)}\right)_{m \times n}=\begin{array}{c|c|c|c|c|} 
& E A_{1} & E A_{2} & \cdots & E A_{n} \\
\hline C D_{1} & x_{11}^{(h)} & x_{12}^{(h)} & \cdots & x_{1 n}^{(h)} \\
\hline C D_{2} & x_{21}^{(h)} & x_{22}^{(h)} & \cdots & x_{2 n}^{(h)} \\
\hline \vdots & \vdots & \vdots & \vdots & \vdots \\
\hline C D_{m} & x_{m 1}^{(h)} & x_{m 2}^{(h)} & \cdots & x_{m n}^{(h)} \\
\hline
\end{array}
$$

where:

$$
\begin{aligned}
& x_{11}^{(h)}=\left\langle\left[a_{11}^{(h)}, b_{11}^{(h)}\right],\left[c_{11}^{(h)}, d_{11}^{(h)}\right]\right\rangle ; \\
& x_{12}^{(h)}=\left\langle\left[a_{12}^{(h)}, b_{12}^{(h)}\right],\left[c_{12}^{(h)}, d_{12}^{(h)}\right]\right\rangle ; \\
& x_{1 n}^{(h)}=\left\langle\left[a_{1 n}^{(h)}, b_{1 n}^{(h)}\right],\left[c_{1 n}^{(h)}, d_{1 n}^{(h)}\right]\right\rangle ; \\
& x_{21}^{(h)}=\left\langle\left[a_{21}^{(h)}, b_{21}^{(h)}\right],\left[c_{21}^{(h)}, d_{21}^{(h)}\right]\right\rangle ; \\
& x_{22}^{(h)}=\left\langle\left[a_{22}^{(h)}, b_{22}^{(h)}\right],\left[c_{22}^{(h)}, d_{22}^{(h)}\right]\right\rangle ; \\
& x_{2 n}^{(h)}=\left\langle\left[a_{2 n}^{(h)}, b_{2 n}^{(h)}\right],\left[c_{2 n}^{(h)}, d_{2 n}^{(h)}\right]\right\rangle ; \\
& x_{m 1}^{(h)}=\left\langle\left[a_{m 1}^{(h)}, b_{m 1}^{(h)}\right],\left[c_{m 1}^{(h)}, d_{m 1}^{(h)}\right]\right\rangle ; \\
& x_{m 2}^{(h)}=\left\langle\left[a_{m 2}^{(h)}, b_{m 2}^{(h)}\right],\left[c_{m 2}^{(h)}, d_{m 2}^{(h)}\right]\right\rangle ; \\
& x_{m n}^{(h)}=\left\langle\left[a_{m n}^{(h)}, b_{m n}^{(h)}\right],\left[c_{m n}^{(h)}, d_{m n}^{(h)}\right]\right\rangle .
\end{aligned}
$$

Given the explanations, new IVIF-MCGDM approach has the following steps:

1) A team of the DMs $\left(T M_{h}\right)$ is formed to find the finest candidate by addressing the evolution factors.

2) A DM's weight is computed by linguistic terms and is then converted into IVIF-numbers:

$$
\begin{aligned}
& \tilde{\xi}^{(h)}=\left\langle\mu^{(h)}(\xi), v^{(h)}(\xi)\right\rangle= \\
& \left\langle\left[\xi_{1}^{(h)}, \xi_{2}^{(h)}\right],\left[\xi_{3}^{(h)}, \xi_{4}^{(h)}\right]\right\rangle .
\end{aligned}
$$

3) Evaluation factors are distinguished for the CDLSP.
4) An evaluation factor's weight by $h$-th DMs is expressed with a linguistic term and is converted into the IVIF number:

$$
\begin{aligned}
& \tilde{\varpi}_{j}^{(h)}=\left\langle\mu_{\omega_{j}}^{(h)}, v_{\omega_{j}}^{(h)}\right\rangle= \\
& \left\langle\left[\omega_{j 1}^{(h)}, \omega_{j 2}^{(h)}\right],\left[\omega_{j 3}^{(h)}, \omega_{j 4}^{(h)}\right]\right\rangle .
\end{aligned}
$$

5) Aggregated IVIF weight of evaluation factor by IVIF-WAA operator (Xu, Chen 2007), IVIFWAA ${ }_{\omega}\left(\tilde{\varpi}_{1}, \tilde{\varpi}_{2}, . ., \tilde{\varpi}_{l}\right)$, is calculated by:

$\tilde{\varpi}_{j}=\left\langle\mu_{\omega_{j}}, v_{\omega_{j}}\right\rangle=$

$\left\langle\left[\omega_{j 1}, \omega_{j 2}\right],\left[\omega_{j 3}, \omega_{j 4}\right]\right\rangle=$

$\left\langle\left[1-\prod_{h=1}^{l}\left(1-\xi_{1}^{(h)} \cdot \omega_{j 1}^{(h)}\right)^{\omega_{h}}\right.\right.$,

$\left.1-\prod_{h=1}^{l}\left(1-\xi_{2}^{(h)} \cdot \omega_{j 2}^{(h)}\right)^{\omega_{h}}\right]$,

$\left[\prod_{h=1}^{l}\left(\xi_{3}^{(h)}+\omega_{j 3}^{(h)}-\xi_{3}^{(h)} \cdot \omega_{j 3}^{(h)}\right)^{\omega_{h}}\right.$,

$\left.\left.\prod_{h=1}^{l}\left(\xi_{4}^{(h)}+\omega_{j 4}^{(h)}-\xi_{4}^{(h)} \cdot \omega_{j 4}^{(h)}\right)^{\omega_{h}}\right]\right\rangle$,

where: $\omega=\left(\omega_{1}, \omega_{2}, \ldots, \omega_{l}\right)^{T}$ can be weight vector, $\omega_{h} \in[0,1]$, and $\sum_{h=1}^{l} \omega_{h}=1$.

6) Candidate's score versus the chosen attribute can be assessed by DMs $\left(x_{i j}^{(h)}\right)$.

7) Fuzzy score matrix is constructed $\left(X^{(h)}\right)$.

8) Aggregated IVIF decision matrix is constructed by opinions of DMs and the IVIF-WAA operator $\operatorname{IVIFWAA}_{\omega}\left(\tilde{x}_{i j}^{(1)}, \tilde{x}_{i j}^{(2)}, \ldots, \tilde{x}_{i j}^{(l)}\right)$ by:

$\tilde{x}_{i j}=\left\langle\mu_{x_{i j}}, v_{x_{i j}}\right\rangle=$

$\left\langle\left[a_{i j}, b_{i j}\right],\left[c_{i j}, d_{i j}\right]\right\rangle=$

$\left\langle\left[1-\prod_{h=1}^{l}\left(1-\xi_{1}^{(h)} \cdot a_{i j}^{(h)}\right)^{\omega_{h}}\right.\right.$,

$\left.1-\prod_{h=1}^{l}\left(1-\xi_{2}^{(h)} \cdot b_{i j}^{(h)}\right)^{\omega_{h}}\right]$,

$\left[\prod_{h=1}^{l}\left(\xi_{3}^{(h)}+c_{i j}^{(h)}-\xi_{3}^{(h)} \cdot c_{i j}^{(h)}\right)^{\omega_{h}}\right.$,

$\left.\left.\prod_{h=1}^{l}\left(\xi_{4}^{(h)}+d_{i j}^{(h)}-\xi_{4}^{(h)} \cdot d_{i j}^{(h)}\right)^{\omega_{h}}\right]\right\rangle$,

where: $\omega=\left(\omega_{1}, \omega_{2}, \ldots, \omega_{l}\right)^{T}$ can be weight vector, $\omega_{h} \in[0,1]$, and $\sum_{h=1}^{l} \omega_{h}=1$. 
9) Weighted aggregated IVIF decision matrix is constructed with addressing factors' weights as below:

$\tilde{P}=\left[\tilde{p}_{i j}\right]_{m \times n}$,

where:

$\tilde{p}_{i j}=\tilde{\varpi}_{j} \otimes \tilde{x}_{i j}=\left\langle\left[\omega_{j 1} \cdot a_{i j}, \omega_{j 2} \cdot b_{i j}\right]\right.$,

$\left.\left[\omega_{j 3}+c_{i j}-\omega_{j 3} \cdot c_{i j}, \omega_{j 4}+d_{i j}-\omega_{j 4} \cdot d_{i j}\right]\right\rangle$.

10) IVIF ideal solutions, defined as $\tilde{P}^{*}$ and $\tilde{P}^{-}$, are determined by:

$\tilde{P}^{*}=p_{j}^{*}=\left\{\tilde{p}_{1}^{*}, \ldots, \tilde{p}_{n}^{*}\right\}^{T}=$

$\left\{\left(\max _{i} \tilde{p}_{i j} \mid j \in B\right),\left(\min _{i} \tilde{p}_{i j} \mid j \in C\right)\right\}^{T}$;

$\tilde{P}^{-}=p_{j}^{-}=\left\{\tilde{p}_{1}^{-}, \ldots, \tilde{p}_{n}^{-}\right\}^{T}=$

$\left\{\left(\min _{i} \tilde{p}_{i j} \mid j \in B\right),\left(\max _{i} \tilde{p}_{i j} \mid j \in C\right)\right\}^{T}$,

where: $B$ can be benefit criteria; $C$ can be cost criteria.

11) IVIF positive-ideal separation matrix $\left(P \tilde{S}^{*}\right)$ and negative-ideal separation matrix $\left(N \tilde{S}^{-}\right)$are defined as follows:

$P \tilde{S}^{*}=\left[p \tilde{s}_{i j}^{*}\right]=$

$\left[\begin{array}{cccc}\tilde{p}_{1}^{*}-\tilde{p}_{11} & \tilde{p}_{2}^{*}-\tilde{p}_{12} & \cdots & \tilde{p}_{n}^{*}-\tilde{p}_{1 n} \\ \tilde{p}_{1}^{*}-\tilde{p}_{21} & \tilde{p}_{2}^{*}-\tilde{p}_{22} & \cdots & \tilde{p}_{n}^{*}-\tilde{p}_{2 n} \\ \vdots & \vdots & \vdots & \vdots \\ \tilde{p}_{1}^{*}-\tilde{p}_{m 1} & \tilde{p}_{2}^{*}-\tilde{p}_{m 2} & \cdots & \tilde{p}_{n}^{*}-\tilde{p}_{m n}\end{array}\right]$

and

$$
\begin{aligned}
& N \tilde{S}^{-}=\left[n \tilde{s}_{i j}^{-}\right]= \\
& {\left[\begin{array}{cccc}
\tilde{p}_{11}-\tilde{p}_{1}^{-} & \tilde{p}_{12}-\tilde{p}_{2}^{-} & \cdots & \tilde{p}_{1 n}-\tilde{p}_{n}^{-} \\
\tilde{p}_{21}-\tilde{p}_{1}^{-} & \tilde{p}_{22}-\tilde{p}_{2}^{-} & \cdots & \tilde{p}_{2 n}-\tilde{p}_{n}^{-} \\
\vdots & \vdots & \vdots & \vdots \\
\tilde{p}_{m 1}-\tilde{p}_{1}^{-} & \tilde{p}_{m 2}-p_{2}^{-} & \cdots & \tilde{p}_{m n}-\tilde{p}_{n}^{-}
\end{array}\right] .}
\end{aligned}
$$

12) Proposed Collective Index (CI) is calculated, with a score function by (Yu et al. 2012), for IVIF ideal separation matrices by the proposed following rela-

$\eta_{i}\left(P \tilde{S}^{*}, N \tilde{S}^{-}\right)=\left(\sum_{j=1}^{n} \frac{S\left(p\left(n \tilde{s}_{i j}^{*}\right)\right.}{S\left(n \tilde{s}_{i j}^{-}\right)}\right)^{\frac{1}{n}}+\tilde{\kappa}_{i j^{\prime}} \forall i,(10)$

where:

$S\left(p \tilde{s}_{i j}^{*}\right)=2+\frac{a_{j}^{*}-\omega_{j 1} \cdot a_{i j}}{1-\omega_{j 1} \cdot a_{i j}}-\frac{c_{j}^{*}}{\omega_{j 3}+c_{i j}-\omega_{j 3} \cdot c_{i j}}+$

$\frac{b_{j}^{*}-\omega_{j 2} \cdot b_{i j}}{1-\omega_{j 2} \cdot b_{i j}}-\frac{d_{j}^{*}}{\omega_{j 4}+d_{i j}-\omega_{j 4} \cdot d_{i j}} ;$

$S\left(n \tilde{s}_{i j}^{-}\right)=2+\frac{\omega_{j 1} \cdot a_{i j}-a_{j}^{-}}{1-a_{j}^{-}}-\frac{\omega_{j 3}+c_{i j}-\omega_{j 3} \cdot c_{i j}}{c_{j}^{-}}+$

$\frac{\omega_{j 2} \cdot b_{i j}-b_{j}^{-}}{1-b_{j}^{-}}-\frac{\omega_{j 4}+d_{i j}-\omega_{j 4} \cdot d_{i j}}{d_{j}^{-}}$, where: the first summation denotes all $j$ for which $S\left(n \tilde{s}_{i j}^{-}\right)>0$ while $\tilde{\kappa}_{i j^{\prime}}$ denote all $j^{\prime}$ for which $S\left(n \tilde{s}_{i j}^{-}\right)=0$.

$\tilde{\kappa}_{i j^{\prime}}$ is calculated by:

$\tilde{\kappa}_{i j^{\prime}}=\left(\max _{j}\left(\frac{S\left(p \tilde{s}_{i j}^{*}\right)}{S\left(n \tilde{s}_{i j}^{-}\right)}\right)\right) \frac{1}{\max _{j} S\left(\tilde{\varpi}_{j}\right)}$,

where:

$S\left(p \tilde{s}_{i j}^{*}\right)=2+\frac{a_{j}^{*}-\omega_{j 1} \cdot a_{i j}}{1-\omega_{j 1} \cdot a_{i j}}-\frac{c_{j}^{*}}{\omega_{j 3}+c_{i j}-\omega_{j 3} \cdot c_{i j}}+$

$\frac{b_{j}^{*}-\omega_{j 2} \cdot b_{i j}}{1-\omega_{j 2} \cdot b_{i j}}-\frac{d_{j}^{*}}{\omega_{j 4}+d_{i j}-\omega_{j 4} \cdot d_{i j}}$;

$S\left(n \tilde{s}_{i j}^{-}\right)=2+\frac{\omega_{j 1} \cdot a_{i j}-a_{j}^{-}}{1-a_{j}^{-}}-\frac{\omega_{j 3}+c_{i j}-\omega_{j 3} \cdot c_{i j}}{c_{j}^{-}}+$

$\frac{\omega_{j 2} \cdot b_{i j}-b_{j}^{-}}{1-b_{j}^{-}}-\frac{\omega_{j 4}+d_{i j}-\omega_{j 4} \cdot d_{i j}}{d_{j}^{-}}$;

$\vartheta_{i}\left(P S^{*}, N S^{-}\right)=\left(\sum_{j=1}^{n} S\left(p \tilde{s}_{i j}^{*}\right)\right)^{\frac{1}{n}}+$

$\left(\sum_{j=1}^{n} \frac{1}{S\left(n \tilde{s}_{i j}^{-}\right)}\right)^{\frac{1}{n}}+\tilde{\chi}_{i j^{\prime}}=$

$\left(\sum_{j=1}^{n} \frac{1}{4}\left(2+\frac{a_{j}^{*}-\omega_{j 1} \cdot a_{i j}}{1-\omega_{j 1} \cdot a_{i j}}-\right.\right.$

$\frac{c_{j}^{*}}{\omega_{j 3}+c_{i j}-\omega_{j 3} \cdot c_{i j}}+\frac{b_{j}^{*}-\omega_{j 2} \cdot b_{i j}}{1-\omega_{j 2} \cdot b_{i j}}-$

$\left.\left.\frac{d_{j}^{*}}{\omega_{j 4}+d_{i j}-\omega_{j 4} \cdot d_{i j}}\right)\right)^{\frac{1}{n}}+$

$\left(\sum_{j=1}^{n} 1 /\left(\frac{1}{4}\left(2+\frac{\omega_{j 1} \cdot a_{i j}-a_{j}^{-}}{1-a_{j}^{-}}-\right.\right.\right.$

$\frac{\omega_{j 3}+c_{i j}-\omega_{j 3} \cdot c_{i j}}{c_{j}^{-}}+\frac{\omega_{j 2} \cdot b_{i j}-b_{j}^{-}}{1-b_{j}^{-}}-$

$\left.\left.\left.\frac{\omega_{j 4}+d_{i j}-\omega_{j 4} \cdot d_{i j}}{d_{j}^{-}}\right)\right)\right)^{\frac{1}{n}}+\tilde{\chi}_{i j^{\prime}} \forall i$,

where: the second summation denotes all $j$ for which $S\left(n \tilde{s}_{i j}^{-}\right)>0$ while $\tilde{\chi}_{i j}$ denote all $j^{\prime}$ for which $S\left(n \tilde{s}_{i j}^{-}\right)=0$.

$\tilde{\chi}_{i j^{\prime}}$ is calculated by:

$\tilde{\chi}_{i j^{\prime}}=\left(\min _{j}\left(S\left(n \tilde{s}_{i j^{\prime}}^{-}\right)\right)\right)^{\max S\left(\tilde{\bar{w}}_{j}\right)}=$

$\left(\min _{j}\left(\frac{1}{4}\left(2+\frac{\omega_{j 1} \cdot a_{i j}-a_{j}^{-}}{1-a_{j}^{-}}-\right.\right.\right.$ 


$$
\begin{aligned}
& \frac{\omega_{j 3}+c_{i j}-\omega_{j 3} \cdot c_{i j}}{c_{j}^{-}}+ \\
& \left.\left.\left.\frac{\omega_{j 2} \cdot b_{i j}-b_{j}^{-}}{1-b_{j}^{-}}-\frac{\omega_{j 4}+d_{i j}-\omega_{j 4} \cdot d_{i j}}{d_{j}^{-}}\right)\right)\right)^{\substack{\max S\left(\tilde{\omega}_{j}\right) \\
j}} .
\end{aligned}
$$

The proposed ranking index is finalized as below:

$$
C I_{i}=\eta_{i}+\vartheta_{i} \text {. }
$$

13) Priority of candidates is determined by the proposed CI. Most suitable candidate can here be distinguished according to smaller amounts of $\eta_{i}$ and $\vartheta_{i}$.

\section{Application of proposed approach}

In an application example, an investor company performed a feasibility study to locate an appropriate crossdocks for logistics network design. Network under study is presented in Figure 1, including several suppliers or providers, cross-docking centers and retailers or customers.

In order to properly address the location selection problem, it is necessary to regard various factors for the CDLSP in the feasibility study. Distinguishing potential centers (candidates) and the most suitable candidate of the one improve the company's outcome in a strategic point of view.

A three-level hierarchy decision process is described for the DMs as shown in Figure 2. In the first level, the overall goal of the CDLSP is depicted at the top level of hierarchy. In the second level, different main criteria are presented to obtain the overall goal in logistics network. Also, cross-docking center locations (candidates) are provided in the third level for the selection process. Following are selected criteria and their descriptions (e.g., Alkhedher 2006; Ou, Chou 2009; Demirel et al. 2010; Özcan et al. 2011):

- cross-docking capacity $E A_{1}$ : in the logistics management via a variety of products, the capacity of a cross-docking center is regarded as an important factor for the operational flow to operate properly;

- expansion possibility $E A_{2}$ : the expansion possibility is related to the architectural and layout factors of cross-docking center location;

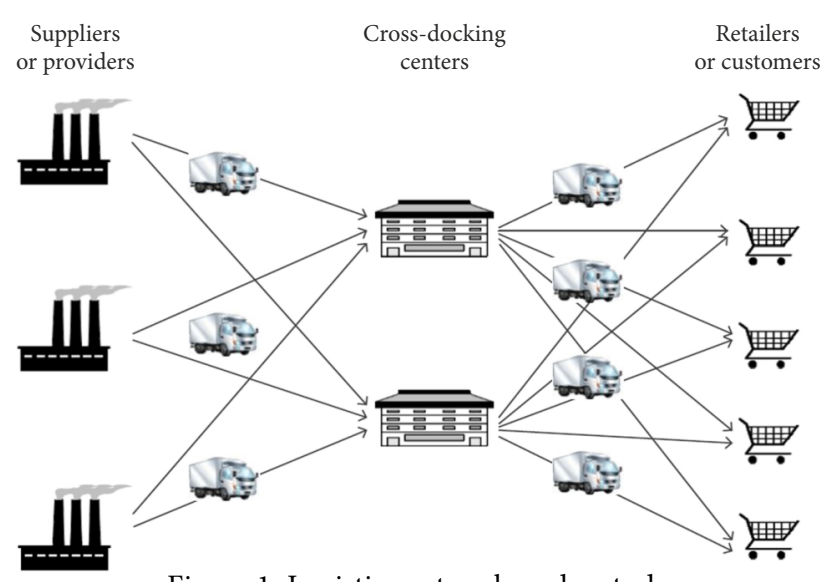

Figure 1. Logistics network under study

- industrial laws and regulations $E A_{3}$ : this factor contains the industrial regulations along with development plans via the macro decision viewpoint;

- proximity to main retailers or customers $E A_{4}$ : considering high demands for products and services, top managers should evaluate a location for the crossdocking center, which is near to main retailers or customers;

- proximity to main suppliers or providers $E A_{5}$ : this factor can be defined as distances of the crossdocking center location to main providers. This help the cross-docking perform the direct movement of containers (trucks) from receiving to shipping with minimum dwell time in between;

- transportation and handling costs $E A_{6}$ : transportation and handling costs have impacts on the crossdocking center location remarkably. Transportation costs can be altered by considering economic approach of regions, and related cross-docking transportation modes. In addition, handling costs contain capital, human resource and equipment.

The relative importance of the chosen evaluation criteria in addition to the experts are depicted by employing the linguistic variables defined in Table 1. Then, performance ratings of the cross-docking candidates versus evaluation factors are presented in Table 2.

A group consisting of three supply chain managers denoted as $T M_{1}, T M_{2}$ and $T M_{3}$ is made to carry out the

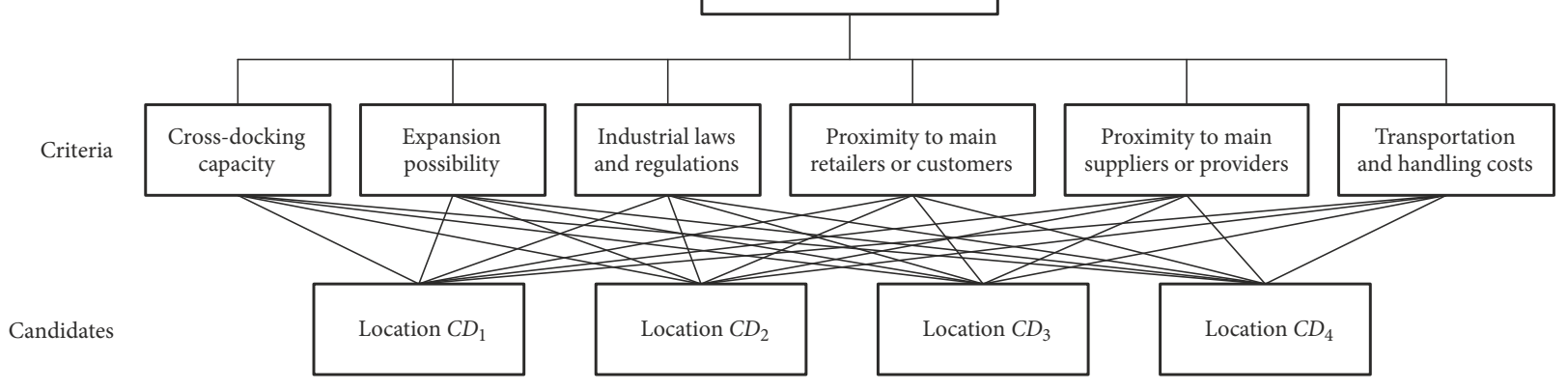

Figure 2. Hierarchical of the CDLP 
assessment and to find the best location for the CDLSP. Since they do not have the same experiences in the company, each one is given a weight that is provided by IVIFnumbers as shown in Table 3.

Table 1. Linguistic variables to express weights of DMs and evaluation factors

\begin{tabular}{|l|c|}
\hline \multicolumn{1}{|c|}{ Linguistic variables } & IVIF numbers \\
\hline Very Important (VI) & $\langle[0.80,0.90],[0.05,0.10]\rangle$ \\
\hline Important (I) & $\langle[0.60,0.75],[0.10,0.20]\rangle$ \\
\hline Medium (M) & $\langle[0.30,0.50],[0.25,0.45]\rangle$ \\
\hline Unimportant (UI) & $\langle[0.20,0.35],[0.45,0.60]\rangle$ \\
\hline Very UnImportant (VUI) & $\langle[0.00,0.10],[0.70,0.90]\rangle$ \\
\hline
\end{tabular}

Table 2. Linguistic variables for values of candidates

\begin{tabular}{|l|c|}
\hline \multicolumn{1}{|c|}{ Linguistic variables } & IVIF numbers \\
\hline $\begin{array}{l}\text { Very Good (VG) / } \\
\text { Very High (VH) }\end{array}$ & $\langle[0.80,0.90],[0.05,0.10]\rangle$ \\
\hline Good (G) / High (H) & $\langle[0.55,0.70],[0.10,0.20]\rangle$ \\
\hline $\begin{array}{l}\text { Medium Good (MG) / } \\
\text { Medium High (MH) }\end{array}$ & $\langle[0.45,0.60],[0.15,0.30]\rangle$ \\
\hline Fair (F) / Medium (M) & $\langle[0.30,0.50],[0.20,0.40]\rangle$ \\
\hline $\begin{array}{l}\text { Medium Bad (MB) / } \\
\text { Medium Low (ML) }\end{array}$ & $\langle[0.25,0.40],[0.35,0.50]\rangle$ \\
\hline Bad (B) / Low (L) & $\langle[0.10,0.30],[0.45,0.60]\rangle$ \\
\hline $\begin{array}{l}\text { Very Bad (VB) / } \\
\text { Very Low (VL) }\end{array}$ & $\langle[0.00,0.10],[0.70,0.90]\rangle$ \\
\hline
\end{tabular}

The six criteria are identified for the CDLSP that are denoted as $E A_{1}, E A_{2}, \ldots, E A_{5}$ and $E A_{6}$. Also, four potential cross-docking center locations (candidates) are denoted as $C D_{1}, C D_{2}, C D_{3}$, and $C D_{4}$. The relative importance of each evaluation factor by team members is expressed and is converted into the IVIF numbers. Then, they are aggregated by the IVIF-WAA, IVIFWAA $\left(_{\omega}\left(\tilde{\varpi}_{1}, \tilde{\varpi}_{2}, \ldots, \tilde{\varpi}_{l}\right)\right.$, by Equation (2) as shown in Table 4.

Fuzzy performance values of alternatives and corresponding IVIF-numbers are assessed by three team members while considering each evaluation criterion. Then, the IVIF rating matrix is presented for each team member as obtained in Table 5. Aggregated matrix is constructed by Equation (3). Obtained values based on the IVIF-WAA operator, IVIFWAA $A_{\omega}\left(\tilde{x}_{i j}^{(1)}, \tilde{x}_{i j}^{(2)}, \ldots, \tilde{x}_{i j}^{(l)}\right)$, are reported in Table 6.

Aggregated IVIF matrix and weights of six evaluation factors are first computed. Then, the weighted IVIF matrix is established and given in Table 7. IVIF ideal solutions are determined according to the benefit criteria for the CDLSP in the application example.

IVIF ideal separation matrixes, $P \tilde{S}^{*}$ and $N \tilde{S}^{-}$, are obtained with Equations (8) and (9) as follows:

$$
\begin{aligned}
P \tilde{S}^{*} & =\left[\begin{array}{llllll}
0.0717 & 0.2125 & 0.1222 & 0.1802 & 0.3129 & 0.1298 \\
0.0000 & 0.1535 & 0.1117 & 0.2565 & 0.2959 & 0.1139 \\
0.0578 & 0.0000 & 0.0000 & 0.0000 & 0.0000 & 0.0000 \\
0.1224 & 0.0151 & 0.0972 & 0.1766 & 0.3129 & 0.0380
\end{array}\right] ; \\
N \tilde{S}^{-} & =\left[\begin{array}{llllll}
0.0550 & 0.0000 & 0.0000 & 0.0955 & 0.0000 & 0.0000 \\
0.1224 & 0.0737 & 0.0121 & 0.0000 & 0.0261 & 0.0180 \\
0.0690 & 0.2125 & 0.1222 & 0.2565 & 0.3129 & 0.1298 \\
0.0000 & 0.2009 & 0.0282 & 0.0992 & 0.0000 & 0.0958
\end{array}\right] .
\end{aligned}
$$

Table 3. Weights of team members

\begin{tabular}{|l|c|c|c|}
\hline Decision-makers (experts) & $T M_{1}$ & $T M_{2}$ & $T M_{3}$ \\
\hline Linguistic variables & $\mathrm{I}$ & $\mathrm{I}$ & $\mathrm{M}$ \\
\hline IVIF numbers & $\langle[0.60,0.75],[0.10,0.20]\rangle$ & $\langle[0.60,0.75],[0.10,0.20]\rangle$ & $\langle[0.30,0.50],[0.25,0.45]\rangle$ \\
\hline
\end{tabular}

Notes: I - Important; M - Medium.

\begin{tabular}{|c|c|c|c|c|c|c|}
\hline $\begin{array}{l}\text { Evaluation factors } \\
\text { (experts) }\end{array}$ & $E A_{1}$ & $E A_{2}$ & $E A_{3}$ & $E A_{4}$ & $E A_{5}$ & $E A_{6}$ \\
\hline$T M_{1}$ & $\mathrm{I}$ & $\mathrm{M}$ & $\mathrm{M}$ & VI & $\mathrm{I}$ & VI \\
\hline$T M_{2}$ & I & $\mathrm{I}$ & I & $\mathrm{I}$ & $\mathrm{I}$ & $\mathrm{I}$ \\
\hline$T M_{3}$ & $\mathrm{M}$ & $\mathrm{I}$ & $\mathrm{M}$ & $\mathrm{I}$ & VI & VI \\
\hline Aggregated IVIF weight & $\begin{array}{l}\langle[0.5180,0.6850], \\
[0.1357,0.2621]\rangle\end{array}$ & $\begin{array}{l}\langle[0.5180,0.6850], \\
[0.1357,0.2621]\rangle\end{array}$ & $\begin{array}{l}\langle[0.4191,0.6031], \\
[0.1842,0.3434]\rangle\end{array}$ & $\begin{array}{l}\langle[0.6825,0.8158], \\
[0.0794,0.1587]\rangle\end{array}$ & $\begin{array}{l}\langle[0.6825,0.8158], \\
[0.0794,0.1587]\rangle\end{array}$ & $\begin{array}{l}\langle[0.7480,0.8643], \\
[0.0630,0.1260]\rangle\end{array}$ \\
\hline
\end{tabular}

Table 4. IVIF importance of evaluation factors

Notes: I - Important; M - Medium; VI - Very Important. 
Table 5. Ratings of cross-docking candidates versus selected criteria

\begin{tabular}{|c|c|c|c|c|}
\hline \multirow{2}{*}{$\begin{array}{l}\text { Evaluation } \\
\text { factors }\end{array}$} & \multirow{2}{*}{$\begin{array}{l}\text { Cross-docking } \\
\text { centers }\end{array}$} & \multicolumn{3}{|c|}{ Decision-makers (experts) } \\
\hline & & $T M_{1}$ & $T M_{2}$ & $T M_{3}$ \\
\hline \multirow{4}{*}{$E A_{1}$} & $C D_{1}$ & $\mathrm{MH}$ & $\mathrm{MH}$ & $\mathrm{MH}$ \\
\hline & $C D_{2}$ & $\mathrm{H}$ & $\mathrm{H}$ & $\mathrm{H}$ \\
\hline & $\mathrm{CD}_{3}$ & $\mathrm{MH}$ & $\mathrm{MH}$ & $\mathrm{H}$ \\
\hline & $C D_{4}$ & M & M & $\mathrm{MH}$ \\
\hline \multirow{4}{*}{$E A_{2}$} & $C D_{1}$ & $\mathrm{ML}$ & $\mathrm{ML}$ & $\mathrm{L}$ \\
\hline & $C D_{2}$ & ML & M & $\mathrm{M}$ \\
\hline & $C D_{3}$ & $\mathrm{H}$ & $\mathrm{H}$ & $\mathrm{MH}$ \\
\hline & $C D_{4}$ & $\mathrm{H}$ & $\mathrm{MH}$ & $\mathrm{H}$ \\
\hline \multirow{4}{*}{$E A_{3}$} & $C D_{1}$ & MB & $\mathrm{G}$ & M \\
\hline & $C D_{2}$ & M & G & MB \\
\hline & $C D_{3}$ & VG & G & $\mathrm{MG}$ \\
\hline & $C D_{4}$ & M & MG & G \\
\hline \multirow{4}{*}{$E A_{4}$} & $C D_{1}$ & $\mathrm{ML}$ & $\mathrm{MH}$ & $\mathrm{H}$ \\
\hline & $C D_{2}$ & ML & $\mathrm{M}$ & $\mathrm{ML}$ \\
\hline & $C D_{3}$ & $\mathrm{H}$ & $\mathrm{VH}$ & $\mathrm{MH}$ \\
\hline & $C D_{4}$ & $\mathrm{MH}$ & M & M \\
\hline \multirow{4}{*}{$E A_{5}$} & $C D_{1}$ & M & $\mathrm{ML}$ & $\mathrm{ML}$ \\
\hline & $C D_{2}$ & ML & M & $\mathrm{M}$ \\
\hline & $C D_{3}$ & $\mathrm{VH}$ & VH & $\mathrm{H}$ \\
\hline & $C D_{4}$ & M & ML & ML \\
\hline \multirow{4}{*}{$E A_{6}$} & $C D_{1}$ & MG & $\mathrm{M}$ & MG \\
\hline & $C D_{2}$ & $\mathrm{MG}$ & MG & $\mathrm{M}$ \\
\hline & $C D_{3}$ & $\mathrm{G}$ & $\mathrm{G}$ & $\mathrm{G}$ \\
\hline & $\mathrm{CD}_{4}$ & G & $\mathrm{G}$ & $\mathrm{F}$ \\
\hline
\end{tabular}

Notes: B - Bad; MB - Medium Bad; VB - Very Bad; F - Fair; G - Good; MG - Medium Good; VG - Very Good; H - High; MH - Medium High; VH - Very High; L - Low; ML - Medium Low; VL - Very Low; M - Medium.
The proposed index (i.e., CI) by values of $\eta_{i}, \vartheta_{i}$ and the score function of the IVIF-numbers are computed by Equations (10)-(13) and shown in Table 8. For instance, for the first candidate $C D_{1}$ we have:

$$
\eta_{1}=\left(\frac{0.0717}{0.0550}+\frac{0.1802}{0.0955}\right)^{\frac{1}{6}}+1.8869 \frac{1}{0.8151}=3.3928
$$

and

$$
\begin{aligned}
& \vartheta_{1}=(0.0717+0.2125+0.1222+ \\
& 0.1802+0.3129+0.1298)^{\frac{1}{6}}+ \\
& \left(\frac{1}{0.0550}+\frac{1}{0.0955}\right)^{\frac{1}{6}}+0.0550^{0.8151}=2.8482 .
\end{aligned}
$$

Thus,

$$
C I_{1}=\eta_{1}+\vartheta_{1}=3.3928+2.8482=6.2410 \text {. }
$$

As given in Table 8, the ranking order of four crossdocking center locations is $C D_{3}, C D_{1}, C D_{4}$ and $C D_{2}$. Eventually, cross-docking center $3 \mathrm{CD}_{3}$ after using the proposed IVIF-MCGDM model is chosen for the CDLSP. Regarding commercial software packages, it is worth to mention that the proposed model for the application example is coded by Visual Basic for Applications (VBA) in the spreadsheet software (i.e., Excel-based VBA macrocode). In addition, it can be performed by MATLAB software for the largesized problems.

\section{Discussion of results}

The results from the application for the CDLSP in this study illustrate that the presented IVIF-MCGDM approach is an adaptable and reasonable one under uncertainty, which helps the committee members to effortlessly

\begin{tabular}{|c|c|c|c|c|c|c|}
\hline Candidates $\begin{array}{c}\text { Evaluation } \\
\text { factors }\end{array}$ & $E A_{1}$ & $E A_{2}$ & $E A_{3}$ & $E A_{4}$ & $E A_{5}$ & $E A_{6}$ \\
\hline$C D_{1}$ & $\begin{array}{l}\langle[0.2275,0.4040], \\
[0.2715,0.4920]\rangle\end{array}$ & $\begin{array}{l}\langle[0.1117,0.2532], \\
[0.4660,0.6548]\rangle\end{array}$ & $\begin{array}{l}\langle[0.1968,0.3706], \\
[0.3160,0.5250]\rangle\end{array}$ & $\begin{array}{l}\langle[0.1968,0.3698], \\
[0.3165,0.5288]\rangle\end{array}$ & $\mid \begin{array}{l}\langle[0.1361,0.2953], \\
[0.3905,0.6093]\rangle\end{array}$ & $\begin{array}{l}\langle[0.1970,0.3780], \\
[0.2879,0.5201]\rangle\end{array}$ \\
\hline$C D_{2}$ & $\begin{array}{l}\langle[0.2790,0.4726], \\
[0.2272,0.4171]\rangle\end{array}$ & $\begin{array}{l}\langle[0.1408,0.3103], \\
[0.3595,0.5935]\rangle\end{array}$ & $\begin{array}{l}\langle[0.2020,0.3807] \\
[0.3010,0.5139]\rangle\end{array}$ & $\begin{array}{l}\langle[0.1361,0.2953], \\
[0.3905,0.6093]\rangle\end{array}$ & $\begin{array}{l}\langle[0.1408,0.3103], \\
[0.3595,0.5935]\rangle\end{array}$ & $\begin{array}{l}\langle[0.2144,0.3901], \\
[0.2806,0.5062]\rangle\end{array}$ \\
\hline$C D_{3}$ & $\begin{array}{l}\langle[0.2366,0.4185], \\
[0.2618,0.4768]\rangle\end{array}$ & $\begin{array}{l}\langle[0.2704,0.4595], \\
[0.2357,0.4304]\rangle\end{array}$ & $\begin{array}{l}\langle[0.3296,0.5237], \\
[0.2153,0.3958]\rangle\end{array}$ & $\begin{array}{l}\langle[0.3296,0.5237], \\
[0.2153,0.3958]\rangle\end{array}$ & $\mid \begin{array}{l}\langle[0.3911,0.5905], \\
[0.1898,0.3528]\rangle\end{array}$ & $\begin{array}{l}\langle[0.2790,0.4726], \\
[0.2272,0.4171]\rangle\end{array}$ \\
\hline$C D_{4}$ & $\begin{array}{l}\langle[0.1653,0.3509], \\
[0.3052,0.5499]\rangle\end{array}$ & $\begin{array}{l}\langle[0.2581,0.4462] \\
[0.2439,0.4460]\rangle\end{array}$ & $\begin{array}{l}\langle[0.2064,0.3932], \\
[0.2776,0.5041]\rangle\end{array}$ & $\begin{array}{l}\langle[0.1833,0.3635], \\
[0.2975,0.5352]\rangle\end{array}$ & $\mid \begin{array}{l}\langle[0.1361,0.2953], \\
[0.3905,0.6093]\rangle\end{array}$ & $\begin{array}{l}\langle[0.2580,0.4469], \\
[0.2435,0.4428]\rangle\end{array}$ \\
\hline
\end{tabular}
use IVIF-numbers to express their uncertain recognition about the degrees that the candidate satisfies and does not satisfy the location evaluation criterion. This study

Table 6. Aggregated IVIF matrix 
Table 7. Weighted aggregated IVIF matrix

\begin{tabular}{|c|c|c|c|c|c|c|}
\hline 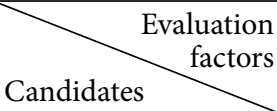 & $E A_{1}$ & $E A_{2}$ & $E A_{3}$ & $E A_{4}$ & $E A_{5}$ & $E A_{6}$ \\
\hline \multirow{2}{*}{$C D_{1}$} & $\langle[0.1178,0.2767]$ & {$[0.0579,0.1734]$} & $\langle[0.0825,0.2235]$ & $\langle[0.1343,0.3017]$ & $\langle[0.0929,0.2409]$ & $\langle[0.1474,0.3267]$, \\
\hline & $[0.3704,0.6251]\rangle$ & $[0.5385,0.7453]\rangle$ & $[0.4420,0.6881]\rangle$ & $[0.3707,0.6036]\rangle$ & $[0.4389,0.6713]\rangle$ & $[0.3327,0.5806]\rangle$ \\
\hline \multirow{2}{*}{$C D_{2}$} & $\langle[0.1445,0.3238]$ & $([0.0729,0.2125]$ & $\langle[0.0847,0.2296]$ & $\langle[0.0929,0.2409]$ & $\langle[0.0961,0.2531]$ & $\langle[0.1603,0.3372]$ \\
\hline & $[0.3321,0.5699]\rangle$ & $[0.4465,0.7000]\rangle$ & $[0.4297,0.6808]\rangle$ & $[0.4389,0.6713]\rangle$ & $[0.4104,0.6580]\rangle$ & $[0.3259,0.5684]\rangle$ \\
\hline \multirow{2}{*}{$C D_{3}$} & $\langle[0.1225,0.2867]$ & {$[0.1401,0.3147]$} & $\langle[0.1381,0.3159]$ & $\langle[0.2249,0.4272]$ & $\langle[0.2669,0.4817]$ & $\langle[0.2087,0.4085]$, \\
\hline & $[0.3620,0.6139]\rangle$ & $[0.3394,0.5796]\rangle$ & $[0.3599,0.6033]\rangle$ & $[0.2776,0.4917]\rangle$ & $[0.2541,0.4555]\rangle$ & $[0.2759,0.4906]\rangle$ \\
\hline \multirow{2}{*}{$C D_{4}$} & $\langle[0.0856,0.2404]$ & {$[0.1337,0.3057]$} & $\langle[0.0865,0.2372]$ & $\langle[0.1251,0.2966]$ & $\langle[0.0929,0.2409]$ & $\langle[0.1930,0.3862]$, \\
\hline & $[0.3995,0.6679]\rangle$ & $0.3465,0.5912]\rangle$ & $[0.4106,0.6744]\rangle$ & $[0.3532,0.6090]\rangle$ & $[0.4389,0.6713]\rangle$ & $[0.2912,0.5130]\rangle$ \\
\hline
\end{tabular}

Table 8. Computational results of the proposed IVIF-MCGDM model

\begin{tabular}{|c|c|c|c|c|}
\hline Candidates & $\eta_{i}$ & $\vartheta_{i}$ & $C I_{i}$ & $\begin{array}{c}\text { IVIF-MCGDM } \\
\text { model ranking }\end{array}$ \\
\hline$C D_{1}$ & 3.3928 & 2.8482 & 6.2410 & 2 \\
\hline$C D_{2}$ & 18.8167 & 3.4252 & 22.2419 & 4 \\
\hline$C D_{3}$ & 0.9711 & 2.4877 & 3.4588 & 1 \\
\hline$C D_{4}$ & 5.5843 & 2.9868 & 8.5711 & 3 \\
\hline
\end{tabular}

successfully introduces the selection process of the crossdocking candidates by evaluation factors from the ordinal fuzzy sets to interval-valued of IFSs' relations in establishing and aggregating the decision by the IVIF matrix. The presented steps show the main finer points of the IVIFMCGDM model over the traditional assessment studies.

The basic principle of traditional TOPSIS method is that the best alternative should consider the shortest distance from the positive-ideal solution and the farthest distance from the negative-ideal solution (Hwang, Yoon 1981; Chen, Hwang 1992). In addition, the extension of TOPSIS method by Chen (2000) with conventional fuzzy numbers was known as the solving decision problems under fuzzy uncertainty.

The proposed IVIF-MCGDM model introduced in this study has the following novelties:

- a new extension of fuzzy sets in IVIF form for the compromise solution under multi-criteria is presented to demonstrate greater adaptability and better portrayal uncertainties than ordinal fuzzy sets due to the way that the proposed model utilizes IVIF numbers. These fuzzy numbers represent degrees that the candidate satisfies and does not satisfy the criterion provided by the DM versus the selected criteria to cope with more imprecise information;

- a novel ranking index (i.e., CI) using IVIF-indices as well as score function has been developed that properly addresses distances of cross-dock candidates versus IVIF ideal solutions;
- two subtraction and division operations on IVIF sets, proposed by Hashemi et al. (2014), are presented for the CDLSP;

- importance of evaluation factors for the CDLSP in IVIF form, that will be profoundly essential in the GDM for complex logistics network problems, is obviously respected with utilizing two novel IVIF measures;

- proposed model constructs the IVIF ideal separation matrices with the new operations between IVIFnumbers to recognize among the candidates in the fuzzy CDLSP better than other past research that could be obtained by Euclidean distances of candidates from two ideal solutions.

\section{Conclusions}

Because of the high multifaceted nature of logistics networks, there are several difficulties for an individual expert to take all the critical parts of choice issues into consideration in real-life situations and applications.

Experts cannot precisely describe their opinions and preferences over the location candidates versus each criterion and the weight of each evaluation criterion under uncertainty due to time pressure or lack of data. In such cases, the assessment process given by a group of experts can be represented as IVIF numbers. Hence, this study has developed a novel IVIF-MCGDM model by concepts of the compromise solution and a team of experts.

In the model, the performance ratings of candidates in terms of evaluation factors and the relative importance factors were linguistic terms, which were denoted by IVIF numbers. IVIF-WAA operator has been used to aggregate experts' opinions. Then, IVIF ideal separation matrices were constructed based on new subtraction and division operations in the fuzzy assessment process. Finally, a new IVIF ranking index has been presented with the score function of two IVIF indices to consider distances of crossdock alternatives from ideal solutions at the same time. 
Proposed IVIF-MCGDM model also stays away from the challenges from developing TOPSIS methods under IVIF uncertainty.

To demonstrate the application of the presented approach, an application example was given for the CDLSP in logistics networks. Hence, the obtained values highlighted that the approach provided experts an effective and practical way to solve logistics network problems by addressing degrees of satisfiability and non-satisfiability under multiple conflicting criteria in an uncertain environment.

To compare with other past research with ordinal fuzzy sets, it is pointed out that the IVIF-MCGDM approach assist the experts with a useful way to handle GDM problems to denote the performance values of each cross-dock candidate along with importance of evaluation factors.

\section{References}

Alkhedher, M. J. E. J. 2006. Dock Design for Automated CrossDocking Container Terminal: PhD Thesis. Purdue University, West Lafayette, IN, US. 194 p. Available from Internet: https://docs.lib.purdue.edu/dissertations/AAI3239750

Atanassov, K. T. 1986. Intuitionistic fuzzy sets, Fuzzy Sets and Systems 20(1): 87-96.

https://doi.org/10.1016/S0165-0114(86)80034-3

Atanassov, K. T. 1994. Operators over interval valued intuitionistic fuzzy sets, Fuzzy Sets and Systems 64(2): 159-174. https://doi.org/10.1016/0165-0114(94)90331-X

Atanassov, K. T.; Gargov, G. 1989. Interval valued intuitionistic fuzzy sets, Fuzzy Sets and Systems 31(3): 343-349. https://doi.org/10.1016/0165-0114(89)90205-4

Bartholdi, J. J.; Gue, K. R. 2004. The best shape for a crossdock, Transportation Science 38(2): 235-244. https://doi.org/10.1287/trsc.1030.0077

Chen, C.-T. 2000. Extensions of the TOPSIS for group decisionmaking under fuzzy environment, Fuzzy Sets and Systems 114(1): 1-9. https://doi.org/10.1016/S0165-0114(97)00377-1

Chen, S.-J.; Hwang, C.-L. 1992. Fuzzy Multiple Attribute Decision Making: Methods and Applications. Springer-Verlag Berlin Heidelberg. 536 p. https://doi.org/10.1007/978-3-642-46768-4

Chen, T.-Y. 2016. An IVIF-ELECTRE outranking method for multiple criteria decision-making with interval-valued intuitionistic fuzzy sets, Technological and Economical Development of Economy 23(3): 416-452.

https://doi.org/10.3846/20294913.2015.1072751

Chen, T.-Y. 2015. IVIF-PROMETHEE outranking methods for multiple criteria decision analysis based on interval-valued intuitionistic fuzzy sets, Fuzzy Optimization and Decision Making 14(2): 173-198.

https://doi.org/10.1007/s10700-014-9195-Z

Demirel, T.; Demirel, N. Ç.; Kahraman, C. 2010. Multi-criteria warehouse location selection using Choquet integral, Expert Systems with Applications 37(5): 3943-3952.

https://doi.org/10.1016/j.eswa.2009.11.022

Hashemi, H.; Bazargan, J.; Mousavi, S. M.; Vahdani, B. 2014. An extended compromise ratio model with an application to reservoir flood control operation under an interval-valued intuitionistic fuzzy environment, Applied Mathematical Modelling 38(14): 3495-3511. https://doi.org/10.1016/j.apm.2013.11.045

Hashemi, S. S.; Razavi Hajiagha, S. H.; Zavadskas, E. K.; Mahdiraji, H. A. 2016. Multicriteria group decision making with
ELECTRE III method based on interval-valued intuitionistic fuzzy information, Applied Mathematical Modelling 40(2): 1554-1564. https://doi.org/10.1016/j.apm.2015.08.011

Hwang, C.-L.; Yoon, K. 1981. Multiple Attribute Decision Making. Methods and Applications: a State-of-the-Art Survey. SpringerVerlag Berlin Heidelberg. 269 p.

https://doi.org/10.1007/978-3-642-48318-9

Jayaraman, V.; Ross, A. 2003. A simulated annealing methodology to distribution network design and management, European Journal of Operational Research 144(3): 629-645. https://doi.org/10.1016/S0377-2217(02)00153-4

Kahraman, C.; Keshavarz Ghorabaee, M.; Zavadskas, E. K.; Onar, S.C; Yazdani, M.; Oztaysi, B. 2017. Intuitionistic fuzzy EDAS method: an application to solid waste disposal site selection, Journal of Environmental Engineering and Landscape Management 25(1): 1-12.

https://doi.org/10.3846/16486897.2017.1281139

Kellar, G. M.; Polak, G. G.; Zhang, X. 2016. Synchronization, cross-docking, and decoupling in supply chain networks, International Journal of Production Research 54(9): 2585-2599. https://doi.org/10.1080/00207543.2015.1107195

Khalaj, M. R.; Modarres, M.; Tavakkoli-Moghaddam, R. 2014. Designing a multi-echelon supply chain network: a car manufacturer case study, Journal of Intelligent \& Fuzzy Systems 27(6): 2897-2914. https://doi.org/10.3233/IFS-141250

Ladier, A.-L.; Alpan, G. 2016. Cross-docking operations: current research versus industry practice, Omega 62: 145-162. https://doi.org/10.1016/j.omega.2015.09.006

Lee, H.-S. 2005. A fuzzy multi-criteria decision making model for the selection of the distribution center, Lecture Notes in Computer Science 3612: 1290-1299.

https://doi.org/10.1007/11539902_164

Li, Y.; Liu, P.; Chen, Y. 2016. Some single valued neutrosophic number Heronian mean operators and their application in multiple attribute group decision making, Informatica 27(1): 85-110. https://doi.org/10.15388/Informatica.2016.78

Liu, P. 2016. Special issue "Intuitionistic fuzzy theory and its application in economy, technology and management", Technological and Economic Development of Economy 22(3): 327335. https://doi.org/10.3846/20294913.2016.1185047

Liu, P.; Li, Y.; Antuchevičienè, J. 2016. Multi-criteria decisionmaking method based on intuitionistic trapezoidal fuzzy prioritised OWA operator, Technological and Economic Development of Economy 22(3): 453-469. https://doi.org/10.3846/20294913.2016.1171262

Maknoon, M. Y.; Soumis, F.; Baptiste, P. 2016. Optimizing transshipment workloads in less-than-truckload cross-docks, International Journal of Production Economics 179: 90-100. https://doi.org/10.1016/j.ijpe.2016.05.015

Makui, A.; Haerian, L.; Eftekhar, M. 2006. Designing a multiobjective nonlinear cross-docking location allocation model using genetic algorithm, Journal of Industrial Engineering International 2(3): 27-42.

Mousavi, S. M.; Tavakkoli-Moghaddam, R.; Siadat, A. 2014a. Optimally design of the cross-docking in distribution networks: heuristic solution approach, International Journal of Engineering: Transactions A: Basics 27(4): 533-544.

Mousavi, S. M.; Vahdani, B. 2017. A robust approach to multiple vehicle location-routing problems with time windows for optimization of cross-docking under uncertainty, Journal of Intelligent \& Fuzzy Systems 32(1): 49-62.

https://doi.org/10.3233/JIFS-151050

Mousavi, S. M.; Vahdani, B.; Tavakkoli-Moghaddam, R.; Hashemi, H. 2014b. Location of cross-docking centers and vehicle 
routing scheduling under uncertainty: a fuzzy possibilisticstochastic programming model, Applied Mathematical Modelling 38(7-8): 2249-2264.

https://doi.org/10.1016/j.apm.2013.10.029

Ou, C.-W.; Chou, S.-Y. 2009. International distribution center selection from a foreign market perspective using a weighted fuzzy factor rating system, Expert Systems with Applications 36(2): 1773-1782. https://doi.org/10.1016/j.eswa.2007.12.007

Özcan, T.; Çelebi, N.; Esnaf, Ş. 2011. Comparative analysis of multi-criteria decision making methodologies and implementation of a warehouse location selection problem, Expert Systems with Applications 38(8): 9773-9779.

https://doi.org/10.1016/j.eswa.2011.02.022

Park, J. H.; Park, I. Y.; Kwun, Y. C.; Tan, X. 2011. Extension of the TOPSIS method for decision making problems under interval-valued intuitionistic fuzzy environment, Applied Mathematical Modelling 35(5): 2544-2556.

https://doi.org/10.1016/j.apm.2010.11.025

Prentkovskis, O.; Erceg, Ž.; Stević, Ž.; Tanackov, I.; Vasiljević, M.; Gavranović, M. 2018. A new methodology for improving service quality measurement: Delphi-FUCOM-SERVQUAL model, Symmetry 10(12): 757.

https://doi.org/10.3390/sym10120757

Ratliff, H. D.; Donadlson, H.; Johnson, E.; Zhang, M. 1998. Schedule-Driven Cross-Docking Network. Technical Report. Georgia Institute of Technology, GA, US.

Razavi Hajiagha, S. H.; Hashemi, S. S.; Zavadskas, E. K. 2013. A complex proportional assessment method for group decision making in an interval-valued intuitionistic fuzzy environment, Technological and Economical Development of Economy 19(1): 22-37. https://doi.org/10.3846/20294913.2012.762953

Razavi Hajiagha, S. H.; Mandiraji, H. A.; Hashemi, S. S.; Zavadskas, E. K. 2015. Evolving a linear programming technique for MAGDM problems with interval valued intuitionistic fuzzy information, Expert Systems with Applications 42(23): 9318-9325. https://doi.org/10.1016/j.eswa.2015.07.067

Rong, L.; Liu, P.; Chu, Y. 2016. Multiple attribute group decision making methods based on intuitionistic fuzzy generalized Hamacher aggregation operator, Economic Computation and Economic Cybernetics Studies and Research 50(2): 211-230.

Ross, A.; Jayaraman, V. 2008. An evaluation of new heuristics for the location of cross-docks distribution centers in supply chain network design, Computers \& Industrial Engineering 55(1): 64-79. https://doi.org/10.1016/j.cie.2007.12.001

Stević, Ž.; Pamučar, D.; Zavadskas, E. K.; Ćirović, G.; Prentkovskis, O. 2017. The selection of wagons for the internal transport of a logistics company: a novel approach based on rough BWM and rough SAW methods, Symmetry 9(11): 264. https://doi.org/10.3390/sym9110264

Vlachopoulou, M.; Silleos, G.; Manthou, V. 2001. Geographic information systems in warehouse site selection decisions, International Journal of Production Economics 71(1-3): 205212. https://doi.org/10.1016/S0925-5273(00)00119-5

Wei, G.; Wang, X. 2007. Some geometric aggregation operators based on interval-valued intuitionistic fuzzy sets and their application to group decision making, in 2007 International Conference on Computational Intelligence and Security (CIS 2007), 15-19 December 2007, Harbin, China, 495-499. https://doi.org/10.1109/CIS.2007.84

Xu, Z. 2007a. Methods for aggregating interval-valued intuitionistic fuzzy information and their application to decision making, Control and Decision 22(2): 215-219.

$\mathrm{Xu}, \mathrm{Z}$. 2007b. Multi-person multi-attribute decision making models under intuitionistic fuzzy environment, Fuzzy Opti- mization and Decision Making 6(3): 221-236. https://doi.org/10.1007/s10700-007-9009-7

Xu, Z.-S.; Chen, J. 2007. Approach to group decision making based on interval-valued intuitionistic judgment matrices, Systems Engineering - Theory \& Practice 27(4): 126-133. https://doi.org/10.1016/S1874-8651(08)60026-5

Xue, Y.-X.; You, J.-X.; Lai, X.-D.; Liu, H.-C. 2016. An intervalvalued intuitionistic fuzzy MABAC approach for material selection with incomplete weight information, Applied Soft Computing 38: 703-713.

https://doi.org/10.1016/j.asoc.2015.10.010

Yan, H.; Tang, S.-L. 2009. Pre-distribution and post-distribution cross-docking operations, Transportation Research Part E: Logistics and Transportation Review 45(6): 843-859.

https://doi.org/10.1016/j.tre.2009.05.005

Yu, D.; Wu, Y.; Lu, T. 2012. Interval-valued intuitionistic fuzzy prioritized operators and their application in group decision making, Knowledge-Based Systems 30: 57-66. https://doi.org/10.1016/j.knosys.2011.11.004

Zadeh, L. A. 1965. Fuzzy sets, Information and Control 8(3): 338-353. https://doi.org/10.1016/S0019-9958(65)90241-X

Zavadskas, E. K.; Antuchevičienė, J.; Razavi Hajiagha, S. H.; Hashemi, S. S. 2014. Extension of weighted aggregated sum product assessment with interval-valued intuitionistic fuzzy numbers (WASPAS-IVIF), Applied Soft Computing 24: 10131021. https://doi.org/10.1016/j.asoc.2014.08.031

Zavadskas, E. K.; Antuchevičienè, J.; Razavi Hajiagha, S. H.; Hashemi, S. S. 2015. The interval-valued intuitionistic fuzzy MULTIMOORA method for group decision making in engineering, Mathematical Problems in Engineering 2015: 560690. https://doi.org/10.1155/2015/560690

Zavadskas, E. K.; Nunić, Z.; Stjepanović, Ž.; Prentkovskis, O. 2018a. A novel rough range of value method (R-ROV) for selecting automatically guided vehicles (AGVs), Studies in Informatics and Control 27(4): 385-394. https://doi.org/10.24846/v27i4y201802

Zavadskas, E. K.; Stević, Ž.; Tanackov, I.; Prentkovskis, O. 2018b. A novel multicriteria approach - rough step-wise weight assessment ratio analysis method (R-SWARA) and its application in logistics, Studies in Informatics and Control 27(1): 97-106. https://doi.org/10.24846/v27i1y201810 